\title{
Clutter Noise Reduction for Phased Array Imaging using Frequency-Spatial Polarity Coherence
}

\author{
Rui Gongzhanga), Anthony Gachagan and Bo Xiao \\ Centre for Ultrasonic Engineering, University of Strathclyde, Glasgow, G1 1XW \\ a)Corresponding author: rui.gongzhang@strath.ac.uk
}

\begin{abstract}
A number of materials used in industry exhibit highly-scattering properties which can reduce the performance of conventional ultrasonic NDE approaches. Moving Bandwidth Polarity Thresholding (MBPT) is a robust frequency diversity based algorithm for scatter noise reduction in single A-scan waveforms, using sign coherence across a range of frequency bands to reduce grain noise and improve Signal to Noise Ratio. Importantly, for this approach to be extended to array applications, spatial variation of noise characteristics must also be considered. This paper presents a new spatialfrequency diversity based algorithm for array imaging, extended from MBPT. Each A-scan in the full matrix capture array dataset is partitioned into a serial of overlapped frequency bands and then undergoes polarity thresholding to generate sign-only coefficients indicating possible flaw locations within each selected band. These coefficients are synthesized to form a coefficient matrix using a delay and sum approach in each frequency band. Matrices produced across the frequency bands are then summed to generate a weighting matrix, which can be applied on any conventional image. A $5 \mathrm{MHz}$ linear array has been used to acquire data from both austenitic steel and high nickel alloy (HNA) samples to validate the proposed algorithm. Background noise is significantly suppressed for both samples after applying this approach. Importantly, three side drilled holes and the back wall of the HNA sample are clearly enhanced in the processed image, with a mean $133 \%$ Contrast to Noise Ratio improvement when compared to a conventional TFM image.
\end{abstract}

\section{INTRODUCTION}

Ultrasonic non-destructive evaluation is widely used in industry to test and evaluate a wide range of materials. A specific problem encountered in the testing of coarse-grained materials, such as concrete, austenitic steels, alloys, is that the target echoes reflected back from the flaws/cracks are often buried by the strong structural noise due to the heterogeneous nature and acoustically scattering properties of these materials. This kind of structural noise, sometimes referred as grain noise or clutter noise, is caused by the inherent properties of the material being inspected and hence, cannot be removed by the traditional time averaging or classical spectral filtering techniques. Lowering the frequency can increase the penetration depth, but also reduces the resolution and the capability of small target detection.

For this reason, many noise reduction techniques have been developed to analyze ultrasonic signals in the presence of highly scattering noise. The most common way to achieve this is through frequency diversity or spatial diversity. Frequency diversity based techniques are widely applied to conventional, single transducer inspection techniques, because of their superior performance on A-scans. One well-known frequency diversity based technique is Split Spectrum Processing (SSP) [1]. SSP is based on the concept that structural noise is usually more sensitive in frequency compared with the flaw echo response and uses multiple filtering and non-linear reconstruction to eliminate structure noise. However, SSP is highly dependent on parameter selection and hence, sensitive to materials properties, especially when the signal to noise ratio (SNR) is low. This is caused by the inconsistent instantaneous phase offset when applied to coarse-grained materials. Take one of the well-known SSP algorithms: polarity thresholding (PT) [2] as an example. PT indicates a target echo when all the filtered frequency channels have an identical sign at certain instant in time. However, when materials have strong frequency dependent scattering and 
attenuation properties, certain frequency channels may have an opposite sign at that time instant, if the bandwidth of its associated filter is not selected properly.

To avoid the effect of parameter sensitivity, algorithms like Optimal Detector (OD) [1] and Fragment Recognition Classifier (FRC) [3] have been proposed. These techniques are model based which means they can adjust themselves by appropriate training. However, high quality training datasets cannot always been acquired. Worse still, sometimes the pre-knowledge of the materials is not available at all.

In the medical imaging area, an algorithm named Moving Bandwidth Minimization (MBM) [4] was developed for the purpose of capturing target echoes with different spectrum characteristics and reducing the grain noise in 'localized frequencies'. The concept of 'moving bandwidth' can also be used in this situation to capture the predominating target flaw echoes at a certain frequency range. This can reduce the effect of frequency dependent scattering and attenuation and solve the phase inconsecutive problem associated with PT.

Hence, a robust frequency diversity based algorithm, Moving Bandwidth Polarity Thresholding (MBPT), was recently devised by the authors [5]. By exploring the phase coincidence within a moving bandwidth across the signal spectrum, MBPT reduces the effect of parameter sensitivity problems while maintaining high performance. MBPT uses a moving bandwidth to partition the spectrum of the processed A-scan signal into several sub-bands, inside which the potential defect is detected when all frequency components have uniform sign. Combining all sub-signals from these sub-bands through averaging gives a probability profile of the potential defect position.

In recent years, phased array transducers have become more commonly used in industry. Phased array transducers have by their nature an advantage to include spatial diversity, since they observe targets from different orientations. Structural noise in coarse-grained materials is not only frequency sensitive, but also spatial sensitive. It can be reduced by observing from different angles and positions, with an accurate and appropriate time delay introduced for each observation. The concept of spatial diversity is not new to array application, with algorithms such as Synthetic Aperture Focusing Techniques (SAFT) widely used. With the possibility of different combinations between array elements, many array based imaging techniques have been developed and outclass the performance of SAFT, such as the classic focused B-scan which is also widely used in industry, and the golden standard imaging algorithm Total Focusing Method (TFM) [6]. Many advanced spatial diversity based algorithm have also been proposed recently, such as Spatially Averaged Sub-Array Correlation Imaging (SASACI) [7] and Correlation for Adaptively Focused Imaging (CAFI) [8].

Based on array imaging applications, there are also advanced techniques using both spatial and frequency diversity that have been developed, such as Spectral Distribution Similarity Analysis (SDSA) [9] and Phase Coherence Imaging (PCI) [10]. SDSA calculates the spectrum similarity of A-scan segments across different transmitting-receiving pairs of array elements, while PCI uses the concept that structure noise has a more random distributed phase compared with target flaw across different A-scans.

MBPT is suitable for A-scan signal processing since it is frequency diversity based. However, phased array probes are increasing being used in many applications and importantly, the spatial diversity approach can be applied to the raw A-scan data. The motivation and aim of this paper is to extend the existing single element MBPT algorithm to accommodate an array imaging application and present a new algorithm that takes benefit from both spatial and frequency information, which has been named Frequency-Spatial Polarity Coherence (FSPC).

\section{METHODOLOGY}

The FSPC achieves clutter noise reduction in images by applying both frequency and spatial diversity. The entire procedure can be described in five steps, as illustrated in the flowchart presented in Fig. 1.

\section{A. Spectrum Partition Approach}

Like MBPT [4], for all A-scan traces in the acquired full matrix capture (FMC) dataset, FSPC firstly divides the A-scan data into a set of sub-signals which contain different frequency bands. This is achieved by bandpass filtering the raw signal or applying a window function to its spectrum. The reason to divide the spectrums of the raw signals into these sub-bands is because the attenuation and scattering of the transmitted ultrasound wave is highly frequency dependent. That means the energy of the target flaw can only partly dominate the frequency range of the received echo spectrum. The aim of this spectrum partitioning is trying to find out the most effective frequency range to process. 


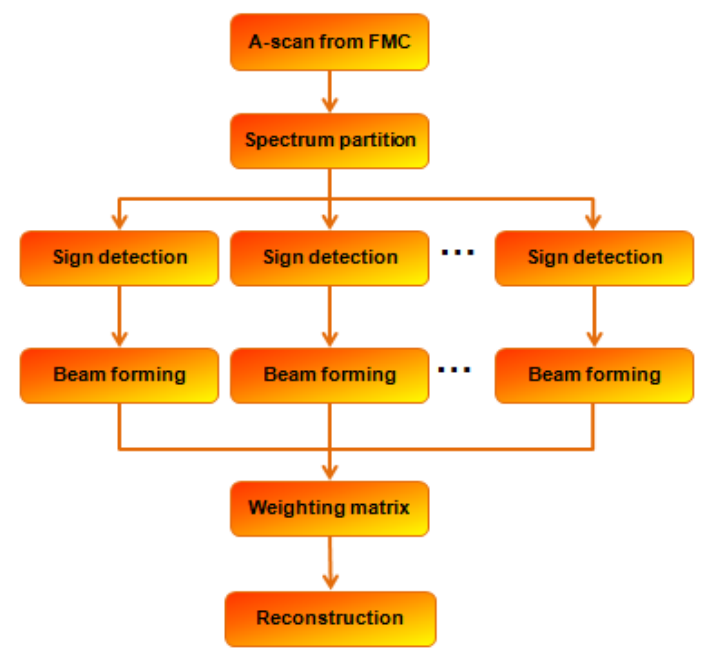

FIGURE 1. Flow chart of Frequency-Spatial Polarity Coherence (FSPC)

To avoid the possibility of failing to detect a flaw, there should be an overlap at least $50 \%$ between adjacent filters/windows. Moreover, to enhance information from the raw A-scan signals, the range of total processed bandwidth which contains all filters or windows, should be chosen as wide as possible: typically around $100 \%$ bandwidth of the transducer central frequency. These sub-signals will be denoted as $Y_{i}(n)$, where $n$ is the time delay and $i$ is the index of filter or window.

\section{B. Sign Detection in Localized Spectrum}

For each sub-signal $Y_{i}(n)$, there is the possibility that the spectrum energy of the target flaw could fully dominate the bandwidth of $Y_{i}(n)$. Hence, the potential location of the target flaw can be detected by applying frequency diversity inside $Y_{i}(n)$ itself. This is achieved by checking the polarity coincidence across different frequency channels inside $Y_{i}(n)$ : Further splitting $Y_{i}(n)$ into several channels within its own bandwidth, if all channels are positive/negative at a specific time delay, then this point is denoted by ' 1 ' or ' -1 ' as a potential flaw location, otherwise a '0' is recorded, as shown below:

$$
Z_{i}(n)=\left\{\begin{array}{l}
1, \text { if all } C_{k}(n)>0 \\
-1, \text { if all } C_{k}(n)<0 \\
0, \text { otherwise }
\end{array}\right.
$$

where $C_{k}(n)$ refers to a channel filtered by a Gaussian window, $k$ is the index of channels.

Based on the principle that the spectrum of a 'real defect' (flaw, back wall etc.) is generally wider than the spectrum of structural noise, the bandwidth of $Y_{i}(n)$ needs to be wide enough to ensure that the algorithm can distinguish the 'real defect' form structural noise. However, for a coarse-grained material, if the bandwidth of $Y_{i}(n)$ is too wide, the spectrum of the 'real defect' may become inconsecutive and hence will no longer predominate. This will cause loss of the detection capability. In this case, the bandwidth of the filters/windows in the previous step is critical for the algorithm performance and typically, this should be around 50\% bandwidth of the transducer central frequency.

\section{Beam Forming in Localized Spectrum}

After all A-scans in the FMC have been processed, a set of coefficient matrices will be generated using all processed $Z_{i}(n)$, based on the desired focal law. Each coefficient matrix represents the polarity coherence of a certain frequency range. 
Generally, the echoes from a 'real defect' has a more consistent phase across A-scans received by different array elements after the focal law has been applied, while structural noise may have a more random distributed phase.

Ideally, the location of a flaw in the matrix will be enhanced due to the sign coincidence across different A-scans, while the structure noise will be suppressed.

\section{Weighting Matrix Generation}

When all coefficient matrices have been calculated, a weighting matrix will then be synthesized through compounding of different frequency bands. This can be achieved in many different ways. A simple way to do this is by averaging all coefficient matrices and setting a suitable threshold. Alternative ways like order statistics and scaled polarity thresholding can also be considered and may provide benefits under certain conditions.

Due to the scattering property of the coarse-grained material, the attenuation is highly frequency dependent. Hence for the region close to the array, more coefficient matrices should be used to increase the imaging resolution, and for the region far from the array, only coefficient matrices in low frequency bands should be included to maintain detection capability. The number of coefficient matrices included for a certain depth depends on the frequency of transmitted signals and the material attenuation property.

\section{E. Reconstruction}

A weighting matrix can be used directly to indicate the defect locations, but this does not contain information on the amplitude associated with the defect response. Multiplying the weighting matrix with an image generated using the same focal law on the same datasets will reduce the background clutter noise while keep the important flaw information. Moreover, a median filter can also be included to smooth the image.

\section{EXPERIMENTAL RESULTS AND DISCUSSION}

\section{A. Results}

To facilitate data collection, experimentally acquired FMC datasets are used here. The FMC is a complete collection of A-scan data from every permutation of transmitting and receiving elements combinations within the array structure. By using FMC, different imaging algorithms can be applied off-line without repeatedly capturing data. Two different samples have been tested to validate the proposed method, using a commercial 5MHz 1-D linear array. A commercial phased array controller (Dynaray, Zetec, Quebec) which is controlled by a MATLAB (The MathWorks, Inc., Natick, MA) script was used to capture the FMC datasets. All images were also post-processed using MATLAB. Details of the samples are provided in Table 1 along with details of the experimental configuration. Images of the austenitic steel samples and the high nickel alloy (HNA) used in this work are shown in Fig. 2.

TABLE 1. Details of experimental parameters

\begin{tabular}{lc}
\hline Experimental parameters & Description \\
\hline Array type & 1-D Linear array (Vermon) \\
Array size & 128 elements \\
Element pitch & $0.7 \mathrm{~mm}$ \\
Center frequency & $5 \mathrm{MHz}$ \\
Fractional bandwidth & $63 \%$ \\
Array controller & Zetec DYNARAY \\
Sampling frequency & $100 \mathrm{MHz}$ \\
Exciting pulse length & $100 \mathrm{nS}$ \\
Tested sample / Wave speed & Austenitic steel $/ 5262 \mathrm{~m} / \mathrm{s}$ \\
& High nickel alloy $(\mathrm{HNA}) / 5900 \mathrm{~m} / \mathrm{s}$ \\
\hline
\end{tabular}




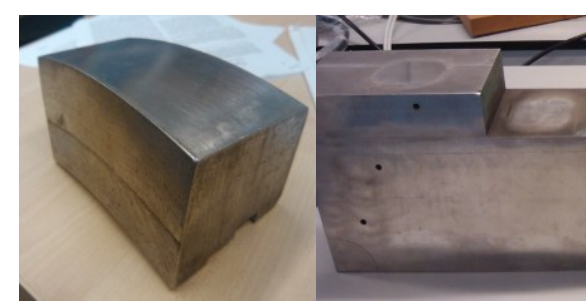

FIGURE 2. Austenitic steel and HNA

Figure 3 presents the FSPC image from the Austenitic steel sample, as well as a classic focused B-scan image for comparison. The images are $140 \mathrm{~mm}$ in depth and contain two reflections of the back wall (located at $54 \mathrm{~mm}$ ) in the sample. The B-scan images were generated by the middle 64 elements of the array, with an aperture size of 16 elements. It can be easily seen that the sample contains strong clutter noise, which reduces the visibility of the first reflection of the back wall and buries the second reflection in the classic focused B-scan. By applying FSPC, both reflections of the back wall are clearly evident and importantly, clutter noise has been reduced.

Figure 4 shows the processed A-scan examples using the classic focused B-scan and the equivalent FSPC techniques (i.e. one of the vertical scan-lines from the images in Fig. 3). Two back wall echoes are successfully detected for the FSPC while only the first reflection of the back wall can be observed in the focused B-scan.

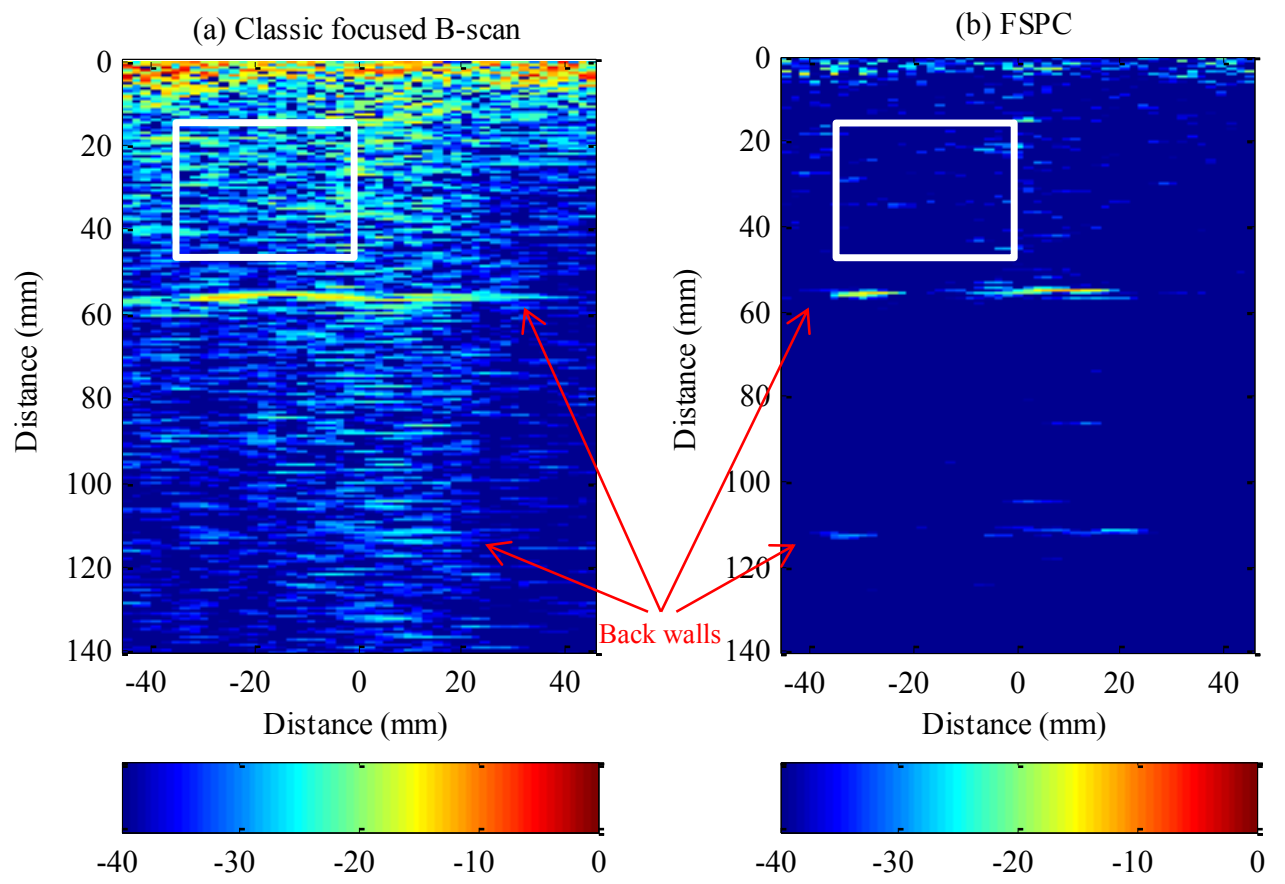

FIGURE 3. Images of the austenitic steel sample

(a) Classic B-scan

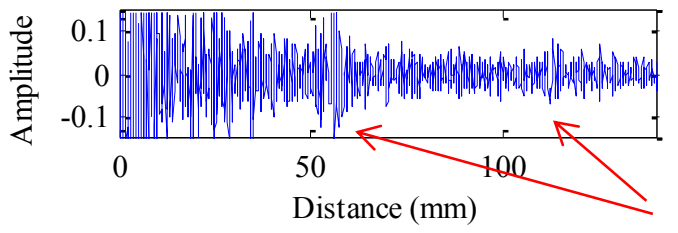

(b) FSPC

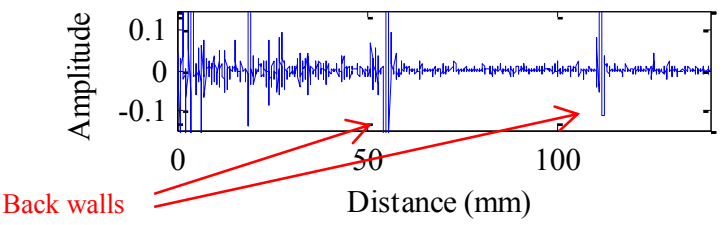

FIGURE 4. Beam formed scan-line of the austenitic steel sample 
Another experiment which compares the performance between TFM and FSPC algorithm is presented in Fig. 5, using the HNA sample. TFM generates images by applying appropriate time delays to each FMC trace to synthesize focusing, and then these contributions are summed together to form a pixel value. The HNA sample is $160 \mathrm{~mm}$ thick and contains 3 side drilled holes (SDH), with depth around of $7 \mathrm{~mm}, 60 \mathrm{~mm}$ and $110 \mathrm{~mm}$ respectively.
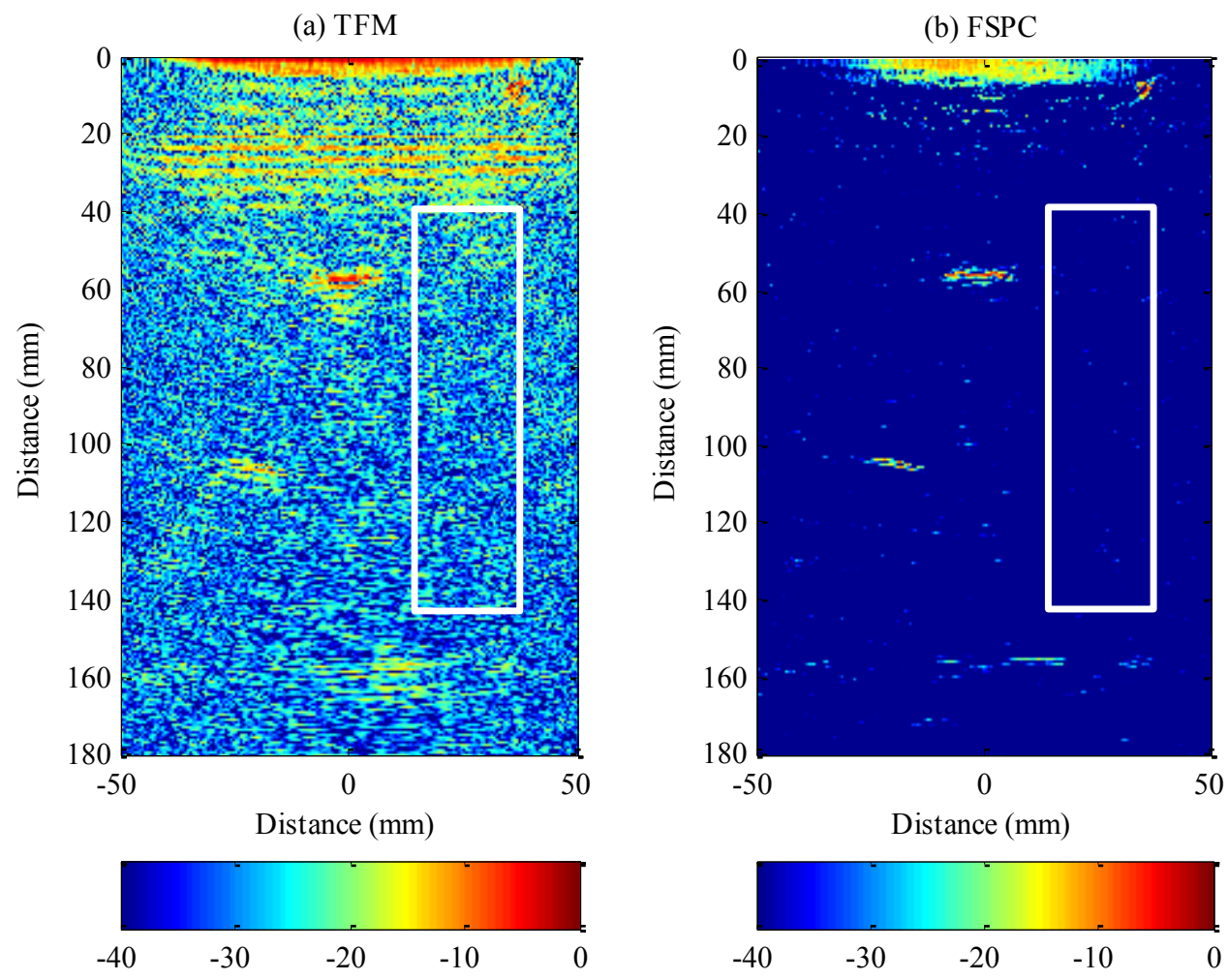

FIGURE 5. TFM and FSPC images of the HNA sample

To show the performance and improvement of FSPC for these experimental results, the related Signal to Noise ratio (SNR) and Contrast to Noise Ratio (CNR) are given in Table 2. The SNR in this case is defined as peak value of the interested area minus average of the noise. The CNR is defined as:

$$
C N R=\frac{A_{s}-\mu_{n}}{\sigma_{n}}
$$

where $A_{s}$ is the peak value of flaw area, $\mu_{\mathrm{n}}$ is the mean value of noise area, $\sigma_{\mathrm{n}}$ is the standard deviation of the speckle area.

The selected noise regions are marked in the figures. From Table 2 it can be seen that FSPC has improved SNR at around $23 \mathrm{~dB}$ for the austenitic steel (average SNR for two reflections of the back wall) and 50dB for the HNA (averaged from three SDHs and the back wall). The average CNR for the three SDHs and the back wall in Fig. 5 has increased by $133 \%$.

TABLE 2. Comparison of SNR and CNR for different imaging algorithms

\begin{tabular}{|c|c|c|c|}
\hline & \multicolumn{2}{|c|}{ SNR (dB) } & \multicolumn{2}{c|}{ CNR } \\
\hline Sample & Austenitic steel & 12 & 3.6 \\
\hline Classic focused B-scan / TFM & 19 & 62 & 8.4 \\
\hline FSPC & 42 & 2 & \\
\hline
\end{tabular}




\section{B. Influence of Frequency Dependent Attenuation}

In course grained materials, the high frequency ultrasound components are more easily scattered and absorbed. Hence, for an imaging region near the array, a wider frequency range could be used for the coefficient matrix to improve the imaging resolution. Figure 6 shows the resolution increases as the processed frequency range is increased. The horizontal axis refers to the total included frequency range, e.g. $3 \mathrm{MHz}$ means all coefficient matrices from $0.5 \mathrm{MHz}$ to $3 \mathrm{MHz}$ are used to generate image. The vertical axis is the number of pixels that higher than $-6 \mathrm{~dB}$, where a lower value refers to a higher resolution. The tested target is the $7 \mathrm{~mm}$ depth $\mathrm{SDH}$, with a $5 \mathrm{~mm}$ diameter, in Fig. 5. Through the inclusion of higher frequencies, the processed images will have higher resolution and detection capability in the region near to the array. In Fig. 6 the resolution stops increasing at around $4 \mathrm{MHz}$ due to frequencies higher than $4 \mathrm{MHz}$ being significant attenuated for this material. Hence coefficient matrices higher than that $4 \mathrm{MHz}$ did not provide much helpful information.

Figure 7 gives in indication of this resolution improvement. It shows the normalized weighting matrix combined using two different set of coefficient matrices. The dark regions in the images refer to the pixels which have a value larger than 0.3: this is an arbitrary threshold chosen to illustrate the influence of the frequency band selection on the performance of the imaging algorithm. Figure 7 (a) only includes a coefficient matrix with the lowest frequency band $(0.5 \mathrm{MHz}-2 \mathrm{MHz}$ ), and Fig. 7 (b) uses all coefficient matrices from $0.5 \mathrm{MHz}$ to $6.5 \mathrm{MHz}$. It can be seen that in Fig. 7 (b) the flaw size is closer to $5 \mathrm{~mm}$ compared with Fig. 7 (a) (as marked on the images), and its shape more closely resembles the spherical surface of the SDH.

For imaging regions far from array, only coefficient matrices with lower frequencies should be used, to increase detection capability. Figure 8 compares the images of the $160 \mathrm{~mm}$ back wall of the HNA sample. The coefficient matrices used in Fig. 8 (a) is $0.5 \mathrm{MHz}-3 \mathrm{MHz}$ while in Fig. 8 (b) it is $1 \mathrm{MHz}-4 \mathrm{MHz}$. It can be clearly seen that the visibility of the back wall is decreased significantly when higher frequencies are included in the FPSC algorithm.

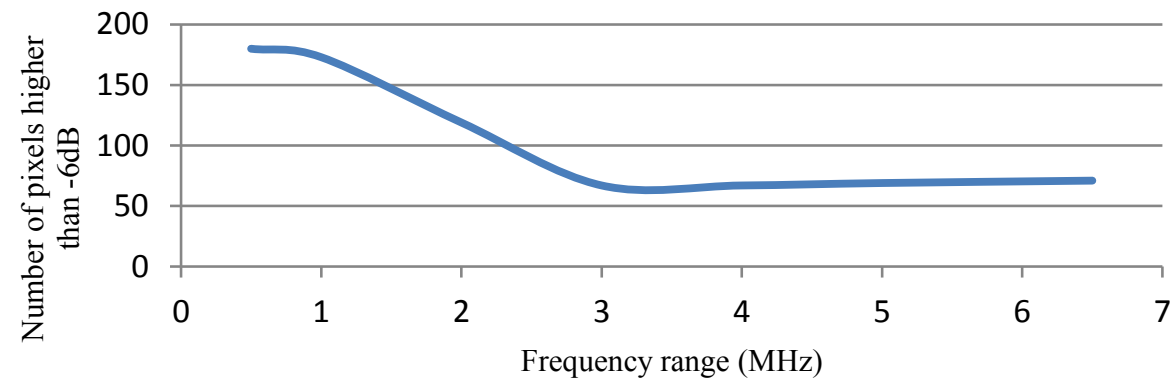

FIGURE 6. The effect of total processed frequency range

(a)

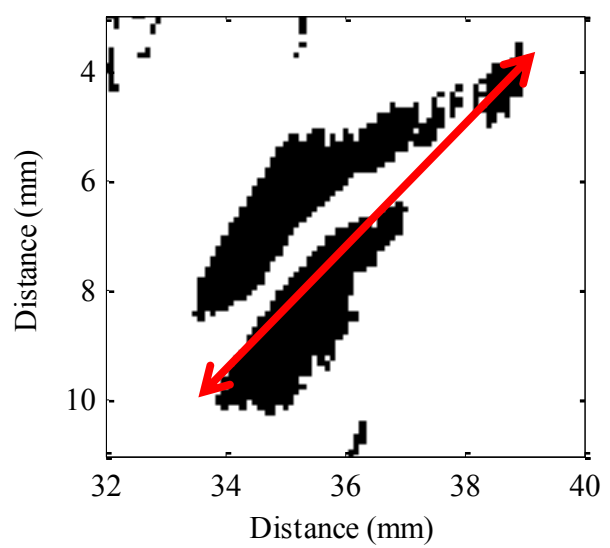

(b)

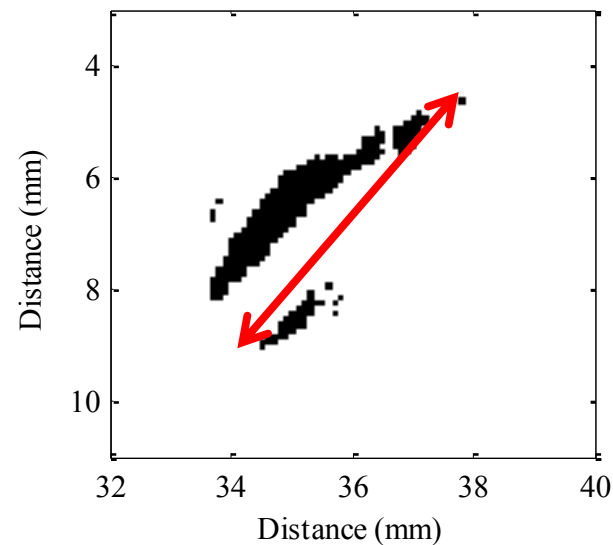

FIGURE 7. Normalized weighting matrices of a SDH with a threshold of 0.3 . (a) with frequency range from $0.5 \mathrm{MHz}$ to $2 \mathrm{MHz}$, (b) with frequency range from $0.5 \mathrm{MHz}$ to $6.5 \mathrm{MHz}$ 
(a)

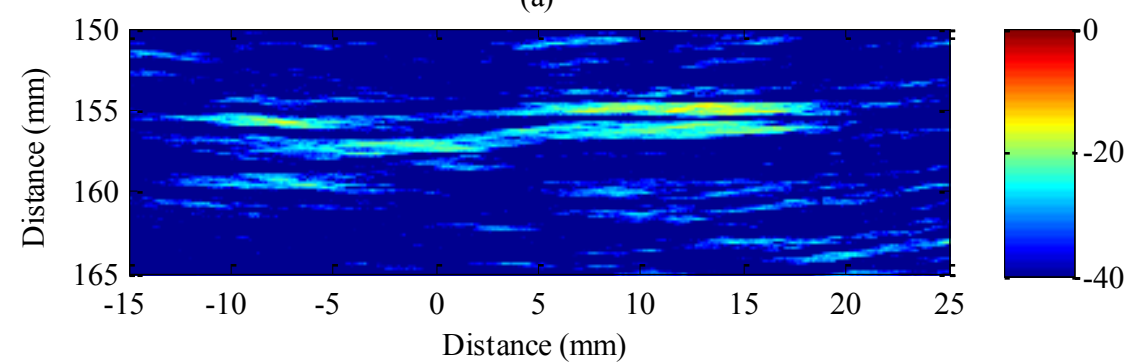

(b)

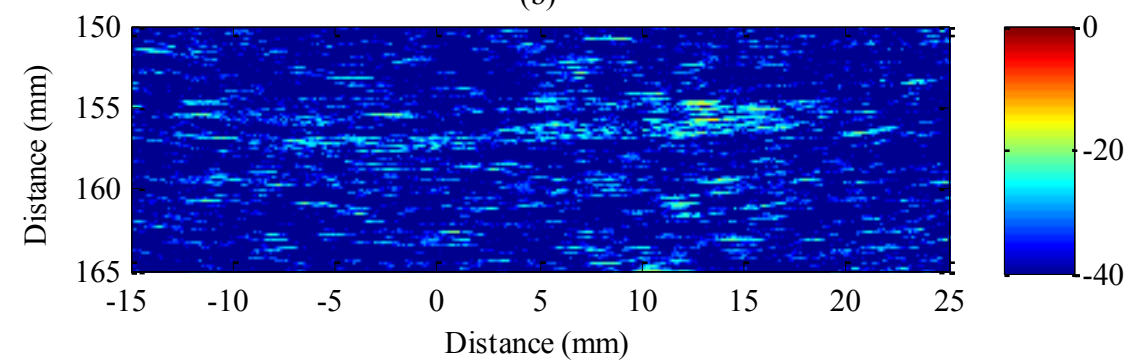

FIGURE 8. FSPC images of back wall of HNA sample. (a) with frequency range from $0.5 \mathrm{MHz}$ to $3 \mathrm{MHz}$, (b) with frequency range from $1 \mathrm{MHz}$ to $4 \mathrm{MHz}$

\section{CONCLUSIONS AND FUTURE WORK}

A new spatial-frequency diversity based algorithm has been proposed for array imaging applications, extended from MBPT. The algorithm explores the polarity coherence among different frequency bands and different A-scan traces, to reduce clutter noise in the image. The proposed algorithm is less sensitive to material properties compared with conventional frequency diversity based techniques. The focal law used to generate the coefficient matrix is the same as used with a conventional imaging algorithm; hence it can be adapted to many imaging applications. In this work, both focused B-scan and TFM have been used. It shows that the FSPC has significantly improved the visibility of the flaw and back wall, compare with focused B-scan and TFM. Future work includes algorithm acceleration and further development of adaptive weighting and reconstruction methods.

\section{ACKNOWLEDGEMENT}

The authors wish to thank E.ON for the supply of samples and for support towards this PhD studentship.

\section{REFERENCES}

1. M. G. Gustafsson, IEEE Transactions on Ultrasonics, Ferroelectrics and Frequency Control, 43, 109-124 (1996).

2. P. M. Shankar, P. Karpur and V. L. Newhouse, IEEE Transactions on Ultrasonics, Ferroelectrics and Frequency Control, 36, 101-108 (1999)

3. R. Gongzhang, M. Li, T. Lardner and A. Gachagan, "Robust Defect Detection in Ultrasonic Non-Destructive Evaluation (NDE) of Difficult Materials" in Ultrasonics Symposium-2012, (IEEE International, Dresden, 2012), pp. 467-470.

4. M. Grevillot, J. Cudel, J. J. Meyer and S. Jacquey, Ultrasonics, 37, 417-422(1999).

5. R. Gongzhang, M. Li, B. Xiao, T. Lardner and A. Gachagan, "Robust frequency diversity based algorithm for clutter noise reduction of ultrasonic signals using multiple sub-spectrum phase coherence," in Review of 
Progress in Quantitative Nondestructive Evaluation-2013, AIP Conference Proceedings 1581, edited by D. E. Chimenti et al. (American Institute of Physics, Baltimore, MA), 2014, pp 1948-1955

6. C. Holmes, B. Drinkwater, and P. Wilcox, NDT \& E International, 38, 701-711 (2005).

7. T. Lardner, M. Li, R. Gongzhang and A. Gachagan, "A New Speckle Noise Suppression Technique Using Cross-correlation of Array Sub-apertures in Ultrasonic NDE of Coarse Grain Materials" in Review of Progress in Quantitative Nondestructive Evaluation-2012, AIP Conference Proceedings 1511, edited by D. O. Thompson et al. (American Institute of Physics, Denver, CO, 2013), pp. 865-871.

8. T. Lardner, M. Li and A. Gachagan, "Using Phase Information to Enhance Speckle Noise Reduction in Ultrasonic NDE of Coarse Grain Materials" in Review of Progress in Quantitative Nondestructive Evaluation2013, AIP Conference Proceedings 1581, edited by D. E. Chimenti et al. (American Institute of Physics, Baltimore, MA, 2014), pp. 1061-1068.

9. J. Camacho and C. Fritsch, IEEE Transactions on Ultrasonics, Ferroelectrics and Frequency Control, 58, 1006$1015(2011)$.

10. B. Xiao, M. Li, R, Gongzhang, R. L. Leary and A. Gachagan, "Image de-noising via spectral distribution similarity analysis for ultrasonic non-destructive evaluation" in Review of Progress in Quantitative Nondestructive Evaluation-2013, AIP Conference Proceedings 1581, edited by D. E. Chimenti et al. (American Institute of Physics, Baltimore, MA), 2014, pp. 1041-1047. 